Pontifícia Universidade Católica $_{\text {Do Rio de Janelro }}$

Agnaldo Cieslak

\title{
Análise Sistemática da Metodologia de Previsão do DGD em Sistemas Ópticos de Alta Capacidade
}

Dissertação de Mestrado

Departamento de EngenhaRIA ELÉtRICA

Programa de Pós Graduação em Engenharia Elétrica 
Pontifícia Universidade Catálica $_{\text {Da }}$

GRAVB.

Agnaldo Cieslak

\section{Análise Sistemática da Metodologia de Previsão do DGD em Sistemas Ópticos de Alta Capacidade}

Dissertação de Mestrado

Dissertação apresentada como requisito parcial para obtenção do título de Mestre pelo Programa de PósGraduação em Engenharia Elétrica da PUC-Rio.

Orientador: Prof. Jean Pierre von der Weid 


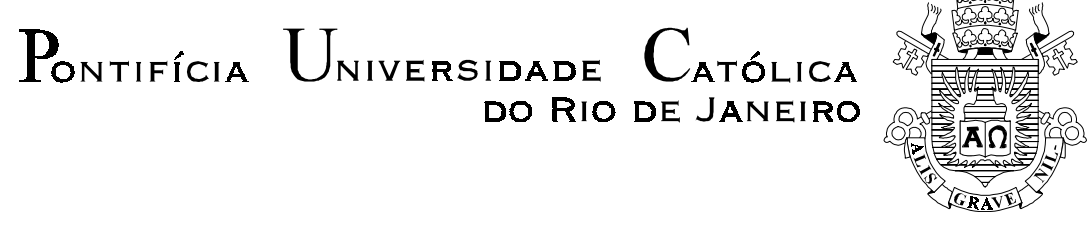

Agnaldo Cieslak

\begin{abstract}
Análise Sistemática da Metodologia de Previsão do DGD em Sistemas Ópticos de Alta Capacidade
\end{abstract}

Dissertação apresentada como requisito parcial para obtenção do grau de Mestre pelo Programa de Pós-Graduação em Engenharia Elétrica do Departamento de Engenharia Elétrica do Centro Técnico Científico da PUC-Rio. Aprovada pela Comissão Examinadora abaixo assinada.

Prof. Jean Pierre von der Weid

Orientador

Centro de Estudos em Telecomunicações - PUC-Rio

Prof. Marcelo Roberto Baptista Pereira Luis Jimenez

Centro de Estudos em Telecomunicações - PUC-Rio

Prof. Luis Carlos Blanco Linares Departamento de Engenharia Mecânica - PUC-Rio

Prof. Rogério Passy

Departamento de Estudos em Telecomunicações - PUC-Rio

Prof. Ney Augusto Dumont Coordenador Setorial do Centro

Técnico Científico - PUC-Rio

Rio de Janeiro, 14 de agosto de 2003 
Todos os direitos reservados. É proibida a reprodução total ou parcial do trabalho sem autorização da universidade, do autor e do orientador.

\section{Agnaldo Cieslak}

Graduou-se em Engenharia Elétrica pelo CEFET-PR (Centro Federal de Educação Tecnológica do Paraná) em 1994. Efetuou intercâmbio e aperfeiçoamento técnico em fibras ópticas no Japão em 1998. Pós graduou-se em Administração em Telecomunicações pela FESP-PR, Latu Sensu em 1999. Participou de congressos internacionais na área de sistemas ópticos. É Engenheiro de Transmissão da Intelig Telecomunicações Ltda, responsável pelo desenvolvimento e soluções de acesso para atendimento a clientes.

Ficha Catalográfica

Cieslak, Agnaldo

Análise sistemática da metodologia de previsão do DGD em sistemas ópticos de alta capacidade / Agnaldo Cieslak ; orientador: Jean Pierre von der Weid. - Rio de Janeiro : PUC, Departamento de Engenharia Elétrica, 2003.

78 f. : il. ; $30 \mathrm{~cm}$

Dissertação (mestrado) - Pontifícia Universidade Católica do Rio de Janeiro, Departamento de Engenharia Elétrica.

Inclui referências bibliográficas.

1. Engenharia elétrica - Teses. 2. DGD. 3. PMD. 4. Probabilidade do DGD. 5. DGD - Metodologia de cálculo. 6. Monte Carlo - Método. 7. Comunicações óticas. 8. Técnicas estatísticas. 9. Metrologia ótica. I. Weid, Jean Pierre van der. II. Pontifícia Universidade Católica do Rio de Janeiro. Departamento de Engenharia Elétrica. III. Título. 
à Danubia, à minha mãe e ao meu pai 


\section{Agradecimentos}

À Deus, fonte da vida.

À Danubia, pelo incentivo constante e severo, e compreensão pelos momentos de abstenção de presença.

Aos meus pais Helena e Albino, pela educação, presença e incentivo recebidos mesmo que à distância.

Ao meu grande amigo João Brito, pelo companheirismo e parceria de mais de 7 anos, seja nos bons ou maus momentos.

Ao Professor Jean Pierre von der Weid, pela discussão, orientação e ensinamentos neste período importante.

À Sra Lourdes, pelo apoio.

À Sônia e Maria Júlia pelo apoio e paciência.

À Ederli e Heverly, Altivir e Altamir que sempre torceram por mim.

À Vice Reitoria Acadêmica pela bolsa parcial oferecida.

À Intelig Telecomunicações Ltda por disponibilizar a base de dados de medidas para as análises realizadas e incentivo financeiro para apresentação do paper no exterior. 


\section{Resumo}

Cieslak, Agnaldo. Análise Sistemática da Metodologia de Previsão do DGD em Sistemas Ópticos de Alta Capacidade. Rio de Janeiro, 2003. 65p. Dissertação de Mestrado - Departamento de Engenharia Elétrica, Pontifícia Universidade Católica do Rio de Janeiro.

Um estudo sistemático da metodologia de determinação da probabilidade do DGD ultrapassar um determinado valor máximo foi apresentado. A metodologia foi discutida sob o ponto de vista teórico, obtendo-se o resultado a partir de uma base de dados recebidos de fabricantes de cabos de fibra óptica. Efetuou-se a comparação com os dados coletados destes mesmos cabos, porém já implantados em backbone.

O resultado deste comparativo sistemático, foi apresentado sob a forma de estatística do valor de DGD ultrapassar o valor máximo estabelecido na norma ITU-T, para sistemas de 10 e 40Gbps.

A partir dos resultados obtidos foi importante a identificação de erros que remontam a origem do processo de fabricação de cabos de fibras ópticas, a restrição na sensibilidade dos equipamentos de medição o que leva a uma superestimação da probabilidade do DGD ultrapassar um certo valor máximo e o próprio conceito do guia TIA/EIA TSB107, que define o método para a obtenção destes resultados.

A criticidade destes resultados pode levar a um erro na escolha de um sistema óptico de transmissão podendo gerar prejuízos muito significativos, sejam financeiro ou temporal.

Foram propostas três recomendações para direcionar a metodologia a uma previsão mais confiável dos resultados obtidos a partir do método 2 TIA/EIA TSB107, gerando mais conforto para o projeto de sistemas de alta capacidade.

\section{Palavras-chave}

DGD; Probabilidade de DGD; Método 2 TIA/EIA TSB107; PMD; IEC; Monte Carlo; Comunicações Ópticas; Metrologia Óptica; Especificação de Enlace 


\section{Abstract}

Cieslak, Agnaldo. Systematical Analysis of the DGD Prediction Methodology in High Capacity Optical Systems. Rio de Janeiro, 2003. 65p. Master Science Thesis - Electrical Engineering Department, Pontifical Catholic University of Rio de Janeiro - Centre for Telecommunication Studies

A systematic study of the methodology that determines the probability of the DGD overcomes a settled maximum value was presented.

The methodology was discussed in a theoretical way; the values were based on a database received from optical fibers manufacturers/cablers. A comparison was conducted taking in consideration these same cables already installed in two backbones.

The result of this systematic comparison was presented, as a statistic of the DGD value exceeds certain maximum value established by the standard ITU-T for systems from 10 to $40 \mathrm{Gbps}$.

Based on these results, the identification of mistakes that ascends the beginning of manufacture process of the optical fiber cables was important. The restriction of the sensibility of measurement devices leads to a overestimation of the probability of the DGD exceeds a given maximum value and the concept of the guideline TIA/EIA TSB107, that defines the methodology of acquisition of these results.

These critical results could lead to a mistake choosing the optical system transmission and cause loss profit as well as time delay in a project.

There were proposed three recommendations in order to guide the methodology for a trustful preview of the obtained results from TIA/EIA method 2, offering more comfort for optical high capacity system design.

\section{Key words}

DGD; DGD Probability; Method 2 TIA/EIA TSB107; PMD; IEC; Monte Carlo; Optical Communications; Optical Metrology; Link Specification 


\section{Sumário}

$\begin{array}{ll}\text { Introdução } & 1\end{array}$

1 Teoria de PMD 4

1.1. Introdução 4

1.2. Origens e Definições da PMD [10] 6

1.3. Efeitos da PMD 10

1.4. Acoplamento de Modos e Estados Principais de Polarização 11

1.5. Caracterização Estatística 13

2 Métodos de Medidas $\quad 18$

2.1. Analisador fixo ou Varredura de comprimento de onda $\begin{array}{ll}\text { (Wavelength Scanning) } & 19\end{array}$

2.1.1. Processo de Medida e Configuração 21

2.1.2. Análise pela Contagem de Extremos 23

2.2. Método Interferométrico 25

3 Teoria da Concatenação e Técnicas Estatísticas 30

3.1. Base de Conhecimentos para Definição dos Métodos de Caracterização 31

3.2. Concatenação pela Média Quadrática 33

3.3. Variação de Maxwell do DGD 34

3.4. Metodologia de Cálculo para PMD de Enlace Óptico 36

3.4.1. Procedimento de Monte Carlo 36

4 Definições e Cálculos para o Método 239

4.1. Definição: $\quad 40$

4.2. Princípio de Cálculo da Convolução 41

4.3. Cálculo da Probabilidade do DGD utilizando a Convolução 42 4.3.1. Aplicando a Técnica de Monte Carlo 43 
5 Estudo Sistemático da Estimativa do DGD através das Técnicas Estatísticas em Comparação com Medidas em Campo 44

5.1. Caracterização do Objeto de Estudo 44

5.2. Avaliação das Probabilidades 49

5.2.1. Roteiro para utilização da norma no cálculo do $P_{D G D} 52$

5.3. Avaliação de enlaces com fabricante $A$

5.4. Avaliação de enlaces com fabricante $B$

5.5. Avaliação de enlaces com fabricante $C \quad 56$

5.6. Avaliações Gerais e Recomendações 57

6 Conclusão 61

7 Glossário 64

8 Referências Bibliográficas 65 


\section{Lista de figuras}

Figura 1 - Efeito da PMD em sistemas de transmissão digital.

Figura 2 - Efeito da PMD em sistemas multicanais 5

Figura 3 - Polarização da luz em fibras $\mathrm{HiBi}$, com lançamento a $45^{\circ}$ com os eixos principais.

Figura 4 - Representação esquemática da propagação de um pulso ao longo de uma fibra $\mathrm{HiBi}$. 8

Figura 5 - Evolução emporal dos estados principais de polarização $\quad 9$ Figura 6 - Esquemático da evolução temporal do DGD entre os dois estados de polarização

Figura 7 - Modelo conceitual de PMD. (a) birrefringência simples. (b) birrefringências concatenadas aleatórias. (c) entrada e saída dos estados principais de polarização.

10

Figura 8 - Variação da polarização de saída com $\lambda$. (a) dispositivo sem acoplamento de modo. (b) fibras SM longas: acoplamento de modos aleatório 13

Figura 9 - Representação do vetor de dispersão da PMD. 14

Figura 10 - Exemplo de distribuições Maxwelliana e Gaussiana. 16

Figura 11 - Espectro de medida de PMD, pelo método de varredura do comprimento de onda para uma fibra $\mathrm{HiBi}$. 20

Figura 12 - Medida de PMD de uma fibra SM normal pelo método de varredura por comprimento de onda. 21

Figura 13 - Exemplos de montagem para a medida de PMD pelo método de varredura por comprimento de onda. 22

Figura 14 - Curva de resposta típica para medida por varredura de comprimento de onda. 22

Figura 15 - Medida interferométrica de PMD. (a) interferômetro básico. (b) montagem do teste. (c) Curva de resposta para birrefringência simples. (d) resposta para modo de acoplamento aleatório. $\quad 26$

Figura 16 - Interferograma obtido com fibra $\mathrm{HiBi}$ na entrada do interferômetro. 
Figura 17 - Interferograma obtido com uma fibra normal na entrada do interferômetro.

Figura 18 - Interferograma obtido com uma fibra normal com baixo acoplamento de modos.

Figura 19 - Interferograma obtido com uma fibra normal com PMD muito baixa

Figura 20 - DGD vs comprimento de onda.

Figura 21 - Função de distribuição de Maxwell.

Figura 22 - Gráfico representativo para determinação de coeficiente de

PMD do enlace pela técnica de Monte Carlo.

Figura 23 - Aproximação adotada para o pior caso de probabilidade da distribuição Maxwell exceder DGD máximo.

Figura 24 - Representação para convolução de duas diracs.

Figura 25 - Universo do coeficiente de PMD e amostragem do fabricante A

Figura 26 - Universo do coeficiente de PMD e amostragem do fabricante $\mathrm{B}$

Figura 27 - Universo do coeficiente de PMD e amostragem do fabricante

C

48

Figura 28 - Probabilidades: comparativo TIA/EIA Medição $x$ Convolução 53

Figura 29 - Probabilidades: comparativo IEC Medição x Convolução 54 Figura 30 - Probabilidades: comparativo TIA/EIA Medição $x$ Convolução 55

Figura 31 - Probabilidades: comparativo IEC Medição x Convolução 56 Figura 32 - Probabilidades: comparativo IEC Medição x Convolução 57 Figura 33 - Probabilidades: comparativo TIA/EIA Medição $x$ Convolução 


\section{Lista de tabelas}

Tabela 1 - Probabilidades baseadas na média dos comprimentos de onda 39

Tabela 2 - Enlaces do backbone \#1 46

Tabela 3 - Enlaces do backbone \#2 